\title{
High-resolution 3D printing of stretchable hydrogel structures using optical projection lithography
}

Puskal Kunwar, Alexander Vincent Struck Jannini, Zheng Xiong, Mark James Ransbottom,

Jamila Shani Perkins, James H. Henderson, Julie M. Hasenwinkel, Pranav Soman*

Syracuse University, Department of chemical and Bioengineering, Syracuse, New York-13244,

USA

*psoman@syr.edu 


\section{Quantification of volume increase during the immersion of DN gel structures in calcium solution.}

The DN gel structure undergoes significant increase in volume after it is immersed to the calcium bath. To quantify the increase in the volume due to absorption of calcium solution, we fabricated DN gel rectangular slab by digital masked based optical lithography. The as-fabricated structure was immersed into the calcium solution and the dimension of the structure is recorded every 24 hrs. Series of photographs show the increase of the volume of the sample before immersing ( $0 \mathrm{hrs})$ and after immersing the structure to $24 \mathrm{hrs}, 48 \mathrm{hrs}$ and $72 \mathrm{hrs}$ to the calcium bath (Figure S1). Photographs show that the most of increment takes place within the $24 \mathrm{hrs}$ of immersion. There is still increment of the volume after 24 hrs of immersion, however the increase in volume is significantly lower compared to that of first $24 \mathrm{hrs}$ immersion. The volume change is quantified in bar diagram, which shows almost 100\% increase in volume in $24 \mathrm{hrs}$. However, in the next $48 \mathrm{hrs,}$ the volume of structure only increases by $\sim 20 \%$.
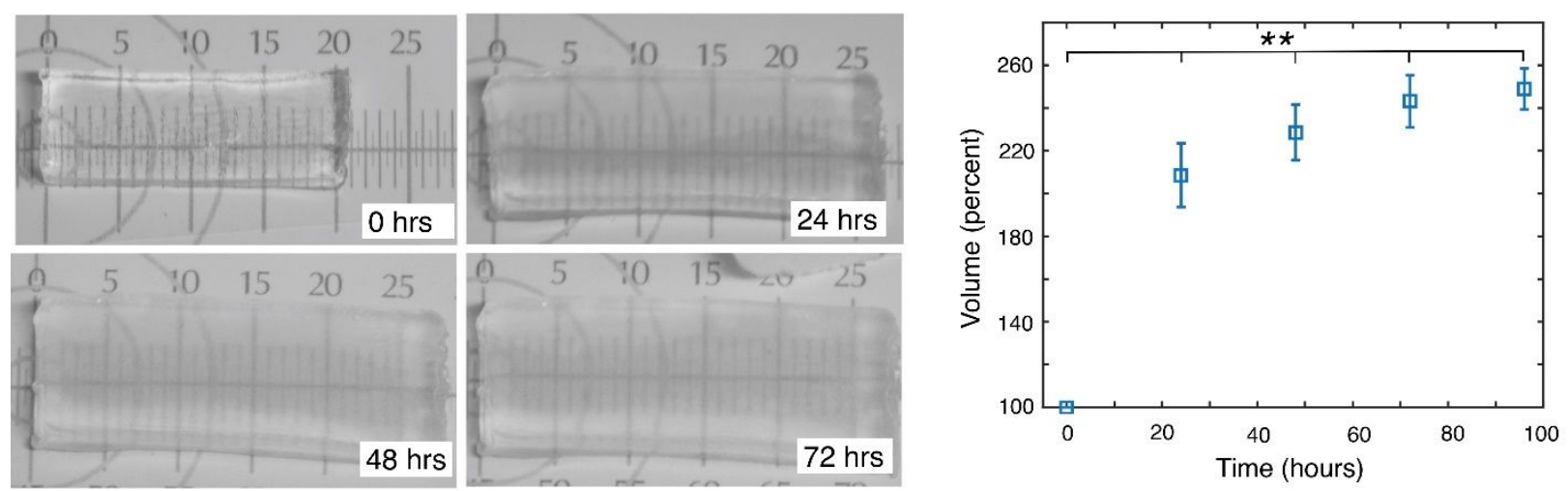

Figure S1. Photographs of the rectangular slab fabricated of DN gel using a digital mask based optical projection lithography. The first photo was taken right after optical fabrication of the structure (0 hrs). The three other photos were recorded after the structure is immersed into the calcium chloride bath for 24 hrs, 48 hrs and 72 hrs respectively. Bar diagram shows quantification of volume increment before and after dipping the fabricated structure to the calcium bath for 
different period of time. ANOVA test showed significant difference among the groups ( ${ }^{* *}$ p value $<0.001)$ (Error bars: Mean $\pm S D$ )

\section{Study of the mechanical properties of fabricated DN gel structures with different fabrication speed and fixed laser power}

The tensile properties of the DN gel structures are studied by fabricating structures with varying scanning speed (also referred as fabrication speed) of $0.045 \mathrm{~mm} / \mathrm{s}, 0.06 \mathrm{~mm} / \mathrm{s}, 0.075 \mathrm{~mm} / \mathrm{s}$ and $0.09 \mathrm{~mm} / \mathrm{s}$ at constant laser intensity of $100 \mathrm{~mW}$ (=laser intensity of $1.65 \mathrm{~mW} / \mathrm{cm}^{2}$ ). A stress vs strain plot was recorded using a tensile tester and is depicted in Figure 6A. The tensile parameters (elastic modulus, strain, and maximum stress) are plotted as a function of fabrication speed (Figure S2).
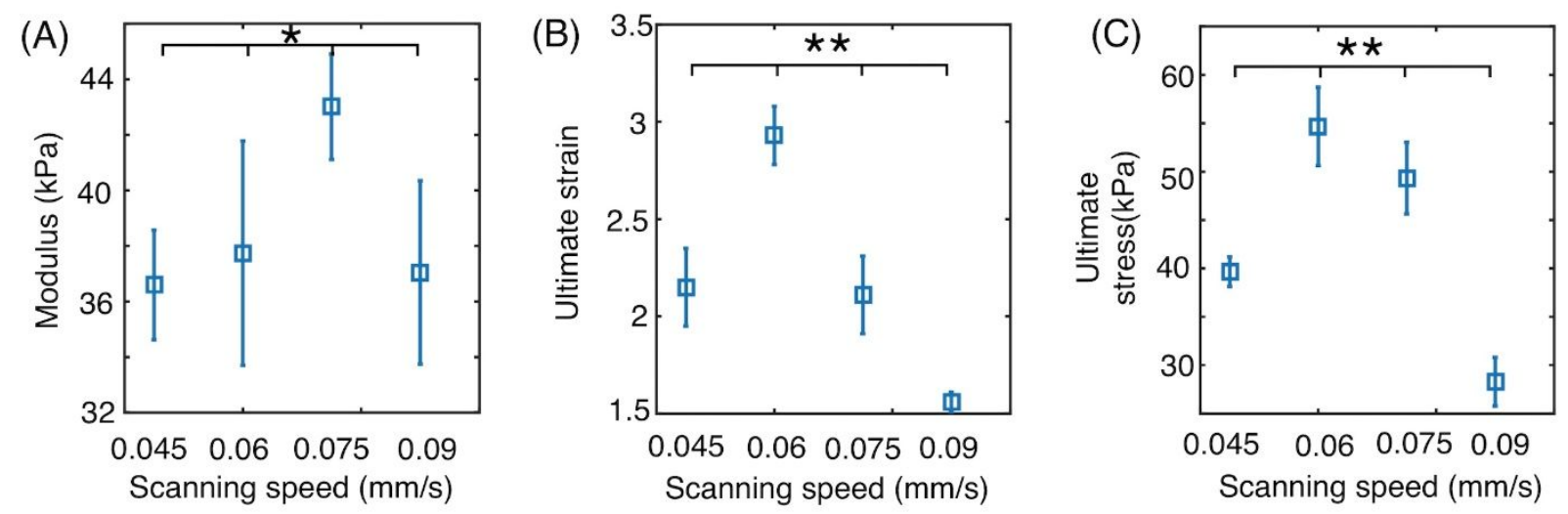

Figure S2. Tensile parameters (A) elastic modulus, (B) strain, and (C) maximum stress are plotted for DN gel structure printed at varying scanning speed and fixed laser power ANOVA test showed significant difference among the groups ( ${ }^{*} p$ value $<0.05,{ }^{* *} p$ value $<0.001$ ) (Error bars: Mean $\pm S D)$ 
